\title{
À propos des stages d'étudiants en entreprise et des pratiques de communication
}

\section{Christian Mesnil}

\section{(2) OpenEdition \\ 12 Journals}

Édition électronique

URL : http://journals.openedition.org/communicationorganisation/1677

DOI : 10.4000/communicationorganisation. 1677

ISSN : $1775-3546$

Éditeur

Presses universitaires de Bordeaux

Édition imprimée

Date de publication : 1 novembre 1993

ISSN : 1168-5549

Référence électronique

Christian Mesnil, «À propos des stages d'étudiants en entreprise et des pratiques de communication », Communication et organisation [En ligne], 4 | 1993, mis en ligne le 26 mars 2012, consulté le 19 avril 2019. URL : http://journals.openedition.org/communicationorganisation/1677 ; DOI : 10.4000/communicationorganisation. 1677

Ce document a été généré automatiquement le 19 avril 2019

(c) Presses universitaires de Bordeaux 


\title{
À propos des stages d'étudiants en entreprise et des pratiques de communication
}

\author{
Christian Mesnil
}

1 Ces quelques lignes extraites d'un court article paru dans le journal l'Entreprise ${ }^{1}$ sont précédées d'un titre à la fois provocateur et significatif : « La France en stage ». Depuis le début des années 90 , ce phénomène semble susciter une prise de conscience nouvelle. Les médias se font l'écho de discours moins unanimes qu'auparavant sur le bien-fondé des stages. S'agit-il d'un simple retour de balancier? Après le profond changement du monde enseignant qui, selon le CNPF, « appelle l'entreprise à son secours " ${ }^{2}$, faut-il voir un retour insidieux des méfiances, des blocages et des conservatismes? Sommes-nous en présence d'autres mutations qui dépasseraient cette dualité traditionnelle? L'évolution actuelle n'est-elle pas liée à de nouvelles situations de communication ou de non communication chez les différents partenaires des stages en entreprise? La présente réflexion voudrait creuser ces questions et observer en particulier ce qui favorise ou au contraire interfère, voire bloque cette communication entre les protagonistes des stages. Elle exclut les stages d'adultes, en dépit de leur nombre très important, pour se consacrer aux stages de jeunes en choisissant délibérément ceux qui relèvent de l'enseignement supérieur. Pourquoi ? Parce que la multiplication des stages semble y avoir généré des situations assez contrastées, voire paradoxales à propos desquelles une analyse globale paraît de plus en plus nécessaire.

2 Pour observer l'ampleur du phénomène et ses caractéristiques, il est indispensable de situer dans un premier temps les stages d'étudiants parmi les autres stages proposés à des jeunes, puis de nous interroger sur le sens même du terme. Ensuite il importe d'examiner pourquoi et selon quelles modalités ces stages sont globalement mis en place avant d'entrevoir la situation et les interrogations actuelles. 


\section{La multiplication des stages d'étudiants en entreprise} extrêmement diversifiées. On ne relève en effet pas moins de onze modalités différentes de séjour en entreprise pour des jeunes de moins de vingt-cinq ans comme en témoigne le panorama ci-dessous ${ }^{3}$. situations rencontrées. ants du secondaire et du supérieur alors qu'elle pourrait elle-même éclater en un tableau à lui seul bien plus complexe que ce récapitulatif. Au-delà du baccalauréat, songeons aux 59 BTS qui comportent un ou plusieurs stages obligatoires répartis sur les deux années de formation ainsi qu'aux 20 spécialités d'IUT qui proposent eux-mêmes des modalités de stage spécifiques à chaque enseignement. Ajoutons les élèves des écoles de gestion et de commerce de niveau $\mathrm{BAC}+2$ ou $\mathrm{BAC}+5$, privées ou reliées aux $\mathrm{CCI}$, les écoles d'ingénieurs, les enseignements professionnalisés des universités (MST, MSG, MIAGE, LEA, DESS) sans oublier les formations nouvellement créées: les IUP, (instituts universitaires professionnalisés qui comportent six mois de stages sur trois années d'étude) et les NFI (nouvelles filières d'ingénieurs) qui imposent parfois deux années de stages sur les cinq années que compte la formation pour les bacheliers.

Ainsi, en se «limitant» à l'enseignement post-baccalauréat qui vient d'être sommairement évoqué, il doit exister plus de deux cents stages obligatoires différents de par leur durée, leur place dans l'année, leurs objectifs, la spécialité, le niveau de formation académique atteint par l'étudiant... Alors que des annuaires d'entreprise comme le KOMPASS, ou France 30000 sont régulièrement mis à jour, aucun répertoire de 
ce type n'existe à ma connaissance pour identifier, classer, présenter les stages postbaccalauréat. Cet outil permettrait par exemple aux entreprises réceptrices de nombreuses demandes de stages de mieux cerner les caractéristiques de ces derniers. Il donnerait un aperçu des périodes de l'année où les émetteurs de demande de stage sont légions pour que chacun des partenaires puissent s'organiser en conséquence.

\section{Quel sens donner au terme « stage »?}

Comment s'y retrouver quant à l'extrême diversité des situations de stage s'ajoute la dilution du sens du terme utilisé ? Certes les dictionnaires ancrent davantage pour le grand public le mot stage dans le sens d'une "Période de formation ou de perfectionnement dans un service d'une entreprise » (Petit Robert). Mais le mot retrouve en réalité un sens - et un fonctionnement ! - plus ancien. "Estage » peut signifier au XII ${ }^{\mathrm{e}}$ siècle "séjour " (que l'on retrouve curieusement dans l'expression parfois utilisée à la place du stage : séjour en entreprise ou séjour d'étude en entreprise). Comme les mots de l'ancien français qui n'ont jamais un sens mais des valeurs suivant le contexte dans lequel ils sont employés, le terme stage présente actuellement un palette de sens dénotés particulièrement riche, chacun dans l'enseignement, dans les médias, dans les entreprises utilise et complète le mot selon un référentiel qui lui est propre.

24 À lui seul, «stage » recouvre en effet trois significations. Il désigne d'abord une période de formation ou de perfectionnement pratique sur le terrain soit pour des étudiants en cours de formation académique, soit pour des étudiants sur le point d'être embauchés, soit pour des employés nouvellement arrivés dans l'entreprise comme c'est le cas chez Renault, Citroën, Péchiney ou Axon ${ }^{4}$. "Stage » rime ensuite pour certaines entreprises avec jobs d'été. «La confusion vient souvent du fait que les jobs d'été, même sans s'inscrire dans un cursus de formation, sont considérés comme très éducatifs lorsqu'ils constituent les premiers contacts d'un jeune avec la vie d'entreprise. De plus, les stages inclus dans les cursus de formation s'effectuant souvent dans la période d'été, il est parfois difficile pour un chef d'entreprise de délimiter les deux réalités » ${ }^{5}$. Enfin « stage » évoque un troisième sens, tout à fait inversé, pour les salariés ou demandeurs d'emploi puisqu'il désigne une période de retour sur les bancs de l'école, un temps de formation théorique et abstraite. Ce sens est également donné à certaines formations académiques dispensées à des étudiants soit sur leur lieu habituel d'enseignement $(\mathrm{c} / \mathrm{f}$ stage de sensibilisation en I.U.T. Génie Chimique) soit sur leur lieu de stage pratique (cf stage théorique d'une à trois semaines pour les étudiants en IUT informatique en stage dans certaines S.S.I.I. ${ }^{6}$.

Le terme stage est ensuite prolongé par une multitude d'expansions. Certaines précisent soit l'activité proposée (marketing, agricole, commercial, électronique) soit le poste occupé (ouvrier, technicien, assistant ingénieur, cadre) soit la mission dévolue (d'observation, de projet d'application, de responsabilité, de recherche, d'étude...). D'autres n'apportent pas vraiment de précision utile sur le stage (stage terrain, pratique, industriel, intégré, en entreprise). D'autres expansions semblent porteuses d'une signification plus pointue, mais elles sont très nombreuses comme en témoignent les exemples suivants: pré-stage, stage d'initiation, de découverte, de connaissance de l'entreprise théorique, d'exécution, de mémoire, de perfectionnement, de rupture, sandwich, de recherche, de qualification, d'intégration, d'insertion, de fin d'étude... La difficulté réside dans l'interprétation de ces expansions. On pressent que des appellations 
différentes recouvrent une réalité sinon semblable du moins fort proche (pré-stage, stage d'initiation, stage de découverte). Mais l'appellation est parfois trompeuse ou source d'ambiguïté. Ainsi le stage de connaissance de l'entreprise peut signifier soit un séjour d'observation, soit un stage ouvrier, soit les deux à la fois. Autre exemple: dans le programme des IUT Information Communication les stages d'application se décomposent en stages d'observation et stages de responsabilité alors que dans l'étude Stages et entreprises ${ }^{7}$, il y a une nette distinction entre les trois appellations.

Chacun sait qu'il est nécessaire pour communiquer de disposer d'un code commun et que celui-ci est constitué d'un ensemble de signes, associations conventionnelles entre signifiant et signifié. Dans notre langue le signifiant stage est associé à de nombreux signifiés. Ces expansions mêmes ne permettent pas toujours de lever l'ambiguïté. Il y a là, à n'en pas douter, un second facteur de dysfonctionnement dans les discours à propos des stages. Il est cependant permis de penser que l'imprécision du terme est compensée par la cohérence des motivations de ceux qui imposent des stages aux étudiants. Voyons si les imperfections du code sont atténuées par l'émission de messages clairs de la part des différents partenaires à propos des stages.

\section{Un consensus superficiel en l'absence de texte de référence}

$27 \mathrm{Au}$ foisonnement quantitatif évoqué initialement s'ajoute un foisonnement terminologique. Les deux difficultés seraient relativement simples à surmonter (répertorier les stages et unifier la terminologie correspondante procèdent d'une même logique) si elles n'étaient pas le signe d'une situation paradoxale: chacun semble intéressé par les stages sans que les règles $\mathrm{du}$ « jeu » soient clairement définies.

Dans l'enseignement supérieur, pour chacun des partenaires, le consensus semble en partie s'expliquer par le flou qui entoure cette notion. "Le rôle des stages dans l'acquisition d'une part, de certains comportements (notamment les exigences de coûts, de délais et de qualité inhérentes à toute activité économique) et d'autre part de savoirs et de savoir-faire techniques fait désormais l'objet d'un large consensus dans la société française " peut-on lire dans le compte rendu du rapport du Haut Comité EducationEconomie sur la professionnalisation des enseignements supérieurs ${ }^{8}$. Le bon sens du vieil adage «C'est au pied du mur qu'on voit le maçon» perce à travers les innombrables messages diffusés dans les brochures et plaquettes de présentation des filières professionnalisées de l'enseignement supérieur. Inclure des stages dans la formation est un argument de vente du produit, un gage de sérieux, de pragmatisme et de professionnalisme. Or, le professionnalisme est aujourd'hui «sans doute l'une des très rares valeurs sur lesquelles notre société s'accorde, toutes générations, couches sociales, régions et professions confondues" observe Yves Cannac, président de l'Institut de l'Entreprise et de la $\mathrm{CEGOS}^{9}$.Cette valeur sociale consensuelle semble avoir entraîné par analogie un autre consensus dont le stage a bénéficié. D’une manière presque magique, un enseignement qui intègrerait des stages prouverait ainsi son efficacité, sa rigueur, son adéquation avec les pratiques et besoins du terrain.

Qu'en est-il en réalité? À cette unanimité globale s'oppose de façon remarquable l'hétérogénéité des calendriers, objectifs et modalités lorsqu'ils existent. En ce qui concerne par exemple les IUP nouvellement créés, il paraît n'exister aucun texte national 
pour justifier préalablement à leur mise en place, les six mois de stages que comportent les trois années de formation menant au diplôme d'«ingénieur-maître ». Pourquoi des stages? Pourquoi six mois? Où les placer dans le cursus? À chaque établissement de produire ses réponses et sa cohérence. Prenons comme second exemple les IUT qui ont été parmi les premiers à prévoir voici vingt-cinq ans des stages obligatoires dans leur formation. Un texte existe, le rapport de la commission ministérielle de 1965. L'évocation des stages y figure de façon très succinte : «En fait il ne semble pas possible d'établir des règles générales valables pour toutes les disciplines». Puisque chacune des vingt spécialités d'IUT est dotée d'un programme national, il est aisé de constater que la moitié d'entre elles n'y mentionne rien à propos des stages et que l'autre les évoque de façon très diversifiée [Entre une ligne et deux pages]. Des habitudes se sont créées suivant les régions ou les spécialités d'IUT. Chacun a (ou n'a pas!) formalisé (ou interprété et complété lorsqu'ils figuraient sur le programme national) les modalités et objectifs du stage qui n'est pas toujours pris en compte de la même manière pour l'obtention du DUT...

Dans l'enseignement post-baccalauréat chaque situation d'alternance a généré de façon plus ou moins explicite ses objectifs, ses contraintes, sa pédagogie propre (quand elle a été formalisée et qu'elle est effectivement appliquée). Comment s'y retrouver ? Des textes existent sur la pédagogie de l'alternance avec des référentiels d'emploi, de compétence, des progressions pédagogiques et des tableaux de liaison, mais ils concernent le niveau $\mathrm{V}$ et plus récemment le niveau IV. Pour les niveaux III $(\mathrm{Bac}+2)$, II et I ( 2 e ou 3 e cycle universitaire et grandes écoles), les seules références nationales actuelles sont juridiques et traitent exclusivement de la convention de stage qui dispense l'entreprise de contrat de travail et de charges sociales puisque le stagiaire garde le statut d'étudiant. Pour le reste chacun procède comme il l'entend (ou se débrouille comme il peut!) et les pratiques les plus variées existent : absence totale de communication entre la période de stage sur le terrain et l'enseignement académique ou interpénétration des modes de formation, stage de quinze jours ou de neuf mois, rémunération inexistante ou indemnisation confortable... On peut lire à ce sujet dans Stages et entreprises « Le manque d'information sur les objectifs est sans cesse regretté (...) Quelle que soit la taille de l'entreprise, un contact étroit avec les programmes de formation des écoles est souhaité, même si dans les pratiques, on semble s'en passer assez facilement ${ }^{10}$.

31 Ce commentaire confirme s'il en était besoin et le déficit d'information entre les partenaires et le fait que les stages en entreprise continuent de fonctionner. Comment et pourquoi l'institution que constituent les séjours en entreprise parvient-elle actuellement à se maintenir?

\section{Le foisonnement des initiatives... et des objectifs}

Les séjours en entreprise semblent pour le moment bénéficier d'une multitude d'initiatives qui tentent de rendre la nébuleuse des stages plus lisible. Conséquence à la fois de ce consensus et de cette absence de repères nationaux, ces initiatives variées, menées par divers partenaires, portent sur différentes modalités de l'alternance.

Nombreux sont les moyens de communication déployés dans ce but. Des brochures et plaquettes sont éditées, des serveurs minitels et banques de données mis en place. Des jumelages, conventions, chartes, accords de partenariat sont signés. Des forums, colloques, tables rondes sont organisés sur l'alternance, la connaissance de l'entreprise, 
l'enseignement professionnalisé. Des services internes ou inter-établissements et des associations proposent leur aide.

À l'origine de ces initiatives se trouvent pratiquement tous les partenaires concernés : des CCI, des unions patronales, des chambres des métiers, des grandes entreprises, des organismes publics, des instituts de recherche, des institutions d'enseignement supérieur, des grandes écoles, des bureaux ou associations d'élèves.

Tantôt il s'agira d'aider les entreprises à distinguer les différents statuts de stagiaires et les obligations administratives correspondantes, tantôt d'établir un calendrier et un tableau comparatif des différents stages obligatoires pour une filière et/ou une région donnée. Parfois l'objectif est de créer des liens privilégiés entre les entreprises et les organismes de formation, de faciliter l'obtention d'un stage par les étudiants ou d'en améliorer le déroulement.

La principale difficulté engendrée par ce foisonnement des initiatives est sa grande hétérogénéité. En effet, il est assez rare que dans un établissement donné les étudiants puissent bénéficier de la conjonction des actions évoquées ci-dessus. Celles-ci sont menées au coup par coup, avec une densité très inégale suivant le dynamisme des partenaires et des régions. En réalité, elles témoignent par ailleurs d'une autre hétérogénéité, celle des utilisations et des points de vue en matière d'alternance.

Certains enseignants considèrent qu'ils passent le relais aux professionnels de l'entreprise pour une activité qui ne relève pas de leur compétence alors que d'autres sont soucieux d'aider leurs étudiants à effectuer un stage enrichissant et/ou complémentaire du programme suivi en cours.

Les étudiants cherchent en priorité soit un site proche d'un domicile, soit une entreprise prestigieuse pour un C.V, soit une rémunération attractive, soit un rythme moins soutenu qu'en formation académique, soit un travail intéressant (mission, matériel, environnement). Mais parfois ils n'ont pas le choix et prennent ce qui se présente après d'innombrables non-réponses ou refus !

Les établissements d'enseignement essaient ainsi de professionnaliser davantage leur formation, de se faire connaître auprès des entreprises (taxe d'apprentissage, formation continue, débouchés pour les futurs diplômés), de donner à leurs étudiants le sens des réalités et une expérience complémentaire ou de libérer des salles de cours et alléger des emplois du temps..

Enfin, par le biais des stages, les entreprises poursuivent également des objectifs variés : se faire connaître, tester des formations académiques, assumer une mission éducative et professionnelle, mais aussi parfois remplacer du personnel à bon compte ou procéder à des recrutements à moindre coût.

41 Chacun des partenaires vise à travers l'alternance un projet qui n'est pas simple et dont certains aspects sont extrêmement pragmatiques. Ces aspects moins glorieux sont rarement mis en avant dans le discours sur les stages. Cette langue de bois, consciente ou inconsciente, contribue à son tour à accentuer les malentendus alors que l'alternance est au cœur d'un processus censé améliorer la communication entre l'Éducation et l'Economie. Le meilleur existe certes, mais en côtoyant parfois le pire, lorsque les partenaires en présence poursuivent des objectifs implicites et divergents. Le stage constitue un espace tout à fait singulier, c'est une sorte de no man's land entre le monde de l'enseignement et celui du travail. L'étudiant relève encore de l'un tout en étant soumis à l'autre. Il n'est plus tout à fait étudiant et n'est pas vraiment employé à part 
entière dans l'entreprise ou l'organisation. La relation qu'il instaure avec le monde du travail et celle qu'il préserve avec son école ou université sont déterminantes pour l'efficacité et la réussite du stage. Comment fonctionnent-elles?

\section{Le stagiaire en communication surveillée} et son milieu. Cette immersion de l'étudiant dans l'entreprise n'est pas aussi facile pour tous. Au-delà du savoir et du savoir-faire, le savoir-être des partenaires joue un rôle déterminant. L'interaction peut aboutir - plus ou moins rapidement - à une relation efficace et chaleureuse. Mais le stagiaire se trouve aussi parfois - de son fait ou non - dans une situation de non-communication, voire d'indifférence à laquelle il ne pourra pas toujours remédier. "Tour les étudiants de niveau supérieur habitués à défendre leur point de vue, à passer les épreuves des jurys les plus sélectifs, il y a quelque chose de paradoxal à ne pas pouvoir se comporter immédiatement en terrain conquis ou à ne pas savoir comment se faire prendre au sérieux dans cet univers nouveau auquel ils se sentent destinés sans y être préparés » peut-on lire dans Stages et Entreprises $»^{11}$. J'ignore s'ils sont très nombreux mais lorsqu'ils existent, les jeunes qui ont trop d'assurance ne sont pas toujours très prisés comme en témoignent ces propos de la Commission progrès des entreprises du CNPF ${ }^{12}$. "Le système de sélection malthusien français - notamment dans nos grandes écoles - a tendance à développer chez certains de nos étudiants une prétention et une arrogance qui témoignent d'une grande immaturité et qui sont à l'opposé des comportements souhaités par les entreprises ». découvrir (ou redécouvrir pour les stagiaires « chevronnés » qui ont parfois jusqu’à huit ou dix séjours en entreprises à effectuer pendant leurs études)? Les spécialistes de gestion des ressources humaines parlent abondamment des méthodes d'analyse et de gestion de compétence. Quand une entreprise recrute, elle cherche un profil correspondant à une fonction particulière. L'étude des situations de travail et la description des emplois et compétences requises pour ceux-ci existent depuis longtemps. Ainsi ne seront jamais confondus en gestion des ressources humaines premier emploi, emploi de remplacement, création de poste et formation. Or le stage procède un peu de ces quatre activités qui nécessitent à la fois expérience, capacité d'adaptation et créativité. La tâche de l'étudiant sera d'autant moins simple que le livret d'accueil conçu dans de grandes entreprises pour faciliter l'insertion du nouvel embauché ne lui sera pas forcément remis... puisqu'un des buts du stage est de lui apprendre à se débrouiller, à chercher seul les informations! L'entreprise n'est d'ailleurs pas la seule responsable de la situation parfois complexe à laquelle le jeune peut être confronté. De la même manière, il existe un singulier décalage entre le laconisme de certains sujets de stage acceptés par les établissements qui dispensent la formation académique et le pointillisme avec lequel les enseignants vont débattre du fond et de la forme des programmes d'enseignement de leur discipline.

Enfin, les étudiants ont de moins en moins le choix. Le nombre des stages prévu dans les formations s'accroît régulièrement et leur durée s'allonge. De plus, les effectifs de l'enseignement supérieur sont en constante progression. De ce fait, la concurrence devient âpre et décrocher un séjour en entreprise peut procéder du parcours du combattant suivant les périodes de l'année et ses spécialités. Le candidat stagiaire se voit 
alors contraint d'accepter toutes les bonnes raisons qui empêchent de cerner au préalable un sujet clair et part ainsi à l'aventure. Il sait que la non obtention du stage remet en cause son passage dans l'année supérieure ou son diplôme. Même si, dès le début, il se rend compte que son séjour en entreprise ne lui apportera pas ce qu'il en attendait, en trouver un autre au "pied levé » sera souvent une gageure. Il ne lui reste plus qu'à attendre une amélioration promise et sans cesse reportée jusqu'au moment où il est trop tard pour réagir ou contacter son établissement.

Alors chacun atténue ou passe sous silence. Le tuteur professionnel surchargé et/ou l'enseignant responsable du suivi (qui découvre tardivement la situation) regrettent, ou minimisent ou considèrent quand même le stage comme acceptable dans leur évaluation finale. À cela s'ajoutent les non-dits du rapport de stage rédigé par l'intéressé. Ils concernent les semaines de désœuvrement, de tâches répétitives ou non adaptées au thème du stage et/ou au niveau de l'étudiant. Il est préférable pour chacun des partenaires d'éviter les conflits et les conséquences ne semblent dramatiques pour personne si ce n'est parfois... le stagiaire. L'étudiant victime d'un stage «bouche-trous » ne diffusera pas l'information urbi et orbi pour au moins quatre raisons. Il peut soit en être responsable par son propre comportement, soit ne pas être en mesure d'apprécier vraiment le niveau du stage, soit ne pas vouloir mettre en difficulté les professionnels dont il dépend et/ou qu'il apprécie par ailleurs, soit

- enfin et surtout ! - voir la non validation de son stage suivie de conséquences plus ou moins lourdes. À cause de cette communication surveillée, cerner avec précision l'ampleur du phénomène n'est pas simple.

47 Expliciter des règles de fonctionnement pour mieux communiquer

48 Les dysfonctionnements en matière de stage, actuellement exacerbés par une conjoncture économique difficile, peuvent de moins en moins être considérés comme des phénomènes marginaux. La fin de l'article du Monde Campus intitulé « Le détournement des stages » montre une prise de conscience au plus haut niveau de la situation.

49 Une évolution souvent perverse qui inquiète au ministère de l'Éducation nationale : « Des réunions sont depuis peu organisées pour répondre à une conjoncture malheureusement porteuse de dérive en matière de stages » explique $t$ - on prudemment à la Direction des Enseignements Supérieurs. Du côté le Haut Comité Education économie vient de créer une commission stages et périodes de formation dans un contexte de forte augmentation de la demande.

50 Il devient en effet urgent de mener pour l'enseignement supérieur une réflexion de fond sur les stages en entreprise. Il ne s'agit pas de le faire dans un esprit polémique ou pour remettre en cause un temps de formation pratique, de passage à l'acte et de mise à l'épreuve profondément original et enrichissant. Il s'agit de penser aux millions de journées de stage imposées chaque année à nos étudiants et aux bénéfices qu'ils sont en droit d'en retirer. Cette réflexion devrait, me semble-t-il, s'articuler prioritairement autour des pôles suivants :

51 - l'équilibre entre l'offre et la demande de stage.

52 - les dérives en matière de séjour d'étude en entreprise.

53 - les aspects économiques et financiers d'une formation sur le terrain.

54 - les difficultés d'une véritable pédagogie de l'alternance. 


\section{NOTES}

1. $\mathrm{N}^{\circ} 71$, septembre 1991

2. La formation des futurs dirigeants synthèse juin 92

3. D'après Stages entreprises p. 195

4. Cf. Partenaires du16 juin 1991 et Usine Nouvelle, janvier 1991.

5. Stages et entreprises Fondation Jeunesse et Entreprises p. 26.

6. SSII société de service en ingenierie de l'Informatique

7. Voir p. 27.

8. Revue Éducation Économie, n 16 , sept. 1992, p. 26

9. Revue Éducation Économie, n¹0, mars 1991, p. 16 (L'entreprise et la formation initiale)

10. Stages et entreprises p. 48

11. Voir p. 103.

12. Synthèse, juin 1992 , p. 14

\section{RÉSUMÉS}

Une des constantes de l'évolution de l'enseignement supérieur en France depuis vingt ans est l'allongement et la multiplication des stages d'étudiants en entreprise. Il semble n'exister aucun descriptif panoramique ou calendrier national mesurant l'ampleur du phénomène. Chacun dans l'enseignement, dans les médias dans l'entreprise, utilise et complète le terme stage à sa guise en l'absence de texte de référence. Le foisonnement d'initiatives et d'objectifs pas toujours convergents des différents partenaires explique la superficialité du consensus qui prévaut pour le moment encore dans la société française. Mais les croissances parallèles des effectifs d'étudiants dans l'enseignement supérieur et des suppressions d'emplois dans les entreprises multiplient actuellement à propos des stages les situations de communication ambiguës.

One of the constant characteristics of the evolution of higher education in France over the past twenty years is the increasing lenght and at the increasing number of professional placement periods for students. There does not seem to be any overall description or national calendar to measure how widespread this phenomenon is. Seeing as there is no official text to refer to. in teaching, in media and in companies, the term "placement period" is used to suit different purposes. The multitude of ideas and alms which are proprosed by the different partners concerned, and which are not always compatible, can explain the superficial consensus which reigns at the moment throughout French society. However, the increase in the number of students in higher education and the parallel reduction in the number of jobs is currently multiplying the number of ambiguous communication situations as far as placement periods are concerned.

«À la banque, à l'hôtel, dans le TGV ou aux Galeries Lafayette, ils (ou elles) sont partout. À croire qu'aujourd'hui la France compte presque plus de stagiaires que de salariés. Et que, remède au chômage, à la «malformation » ou au mal de vivre, le 
stage est devenu la réponse universelle. (...). Finie la grande abstraction scolaire ou professionnelle. Tout le monde sur le terrain.».

\section{AUTEUR}

\section{CHRISTIAN MESNIL}

Christian Mesnil est maître de conférences en techniques de communication à

l'Université du Littoral (Dunkerque, Calais, Boulogne). Il coordonne par ailleurs l'équipe d'enseignants en expression communication de l'ENIC (école nouvelle d'ingénieurs en technologie de la communication) à Villeneuve d'Ascq et fait partie du groupe de recherche en communication des organisations de l'Université de Bordeaux 3. La présente étude tire sa matière de nombreuses visites et soutenances de stages effectuées dans le Nord-Pas de Calais entre 87 et 93. Celles-ci concernent des étudiants de l'IUT du Littoral et des élèves de l'ENIC qui bénéficient d'une alternance où les stages successifs représentent deux des cinq années de formation. 